\title{
More power from water
}

\author{
Competitive technologies to produce electricity through the direct interaction of water with certain materials are \\ now emerging.
}

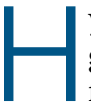
ydropower, in which water flow generates useful work, has been used for more than 2,000 years. Since the late nineteenth century, engineers have generated electricity by converting the kinetic energy of flowing water. Today, 17 per cent of global electricity production still comes from hydropower. Hydropower is, in principle, a sustainable form of energy because of the abundance of the resource and its renewability, but it usually requires big construction projects (dams and reservoirs, for example) that may negatively affect local communities and the environment.

An alternative, though less explored, form of hydropower is based on harnessing osmotic energy by using pressure-retarded osmosis or reverse electrodialysis. Although these approaches have been attracting considerable attention for some decades at a research level, their relatively high costs and low efficiency still inhibit their commercial development. However, in the past few years, new nanomembranes have been found to be capable of generating voltage effectively when an electrolyte solution flows through narrow channels driven by a pressure gradient. For example, Siria et al. reported membranes made of boron nitride nanotubes that can produce power with a density of several kilowatts per square metre $^{1}$; and Feng et al. reported a power density as high as $10^{3} \mathrm{~kW} \mathrm{~m}^{-2}$ when using $\mathrm{MoS}_{2}$ nanomembranes ${ }^{2}$.

Surface charges play a key role in achieving such osmotic energy conversion, and this can be explained by the fact that charged solid surfaces induce the formation of an electric double layer at the interface with aqueous solutions. The effect can be modulated by other surface properties, including surface structures of the materials, surface friction and the substrate hydrophobicity. Because water in these cases is flowing, playing with fluid dynamics by designing specific surface morphologies and chemistries can help to maximize the osmotic energy harvesting, as explained by Siria et al. in their Review ${ }^{3}$.

Aside from osmotic energy, it is also possible to convert thermal and chemical energy into electricity, for example in the evaporation-induced ${ }^{4}$ and moistureinduced electricity generation processes ${ }^{5}$, respectively. In a Review published in this issue of Nature Nanotechnology, Zhang et al. explore a range of phenomena, which they refer to as 'emerging hydrovoltaic effects', in which the electricity is generated by the direct interaction between the materials and water ${ }^{6}$. On properly designed nanostructures, water can be forced to flow, or create waves or drops, or evaporate naturally.

The most interesting aspect of the evaporation and moisture-induced approaches is that no mechanical inputs are needed in the processes. The electric voltage generated in a centimetre-sized carbon black sheet under ambient evaporation condition can be up to $1 \mathrm{~V}$ (ref. ${ }^{4}$ ). To improve these technologies and make them ultimately viable, however, greater effort should be devoted to clarifying the electricity generation mechanism.

Published online: 6 December 2018 https://doi.org/10.1038/s41565-018-0340-7

References

1. Siria, A. et al. Nature 494, 455-458 (2013).

Feng, J. et al. Nature 536, 197-200 (2016)

3. Siria, A. et al. Nat. Rev. Chem. 1, 0091 (2017).

4. Xue, G. et al. Nat. Nanotech. 12, 317-321 (2017).

5. Zhao, F., Liang, Y., Cheng, H., Jiang, L. \& Qu, L. Energy Environ. Sci. 9, 912-916 (2016).

6. Zhang, Z. et al. Nat. Nanotech. https://doi.org/10.1038/s41565018-0228-6 (2018).

\section{A note on the Lena image}

We are asking authors to use alternatives.

W e would like to let our authors, reviewers and readers know that, with immediate effect, we no longer consider submissions containing the Lena (sometimes 'Lenna') image. This decision was taken in consultation with relevant journal editors and affects all Nature Research journals.

A head crop of a picture of Lena Söderberg, which first appeared in November 1972 in the magazine Playboy, has been consistently used as a standard picture in research papers reporting on image processing. In recent years, however, the image has fallen from popularity, with several societies (including the Optical Society and the Society for Industrial and Applied Mathematics) now discouraging its use. We believe that the history of the Lena image clashes with the extensive efforts to promote women undertaking higher education in science and engineering and therefore have decided to adopt this policy.

Authors working in nanophotonics are therefore urged to use alternative images such as 'Cameraman, 'Mandril' or 'Peppers' that have comparable if not superior scientific value. Like the Lena image, all these alternatives are widely available. Useful information about these alternatives can be found in "On alternatives to Lenna" (J. Mod. Opt. 64, 1119-1120; 2017).

In the rare case in which the Lena image becomes necessary to prove the main claim of a manuscript, we reserve the right to consider such a submission, provided that a convincing reason is given by the authors in their cover letter.

Published online: 6 December 2018 https://doi.org/10.1038/s41565-018-0337-2 